\title{
Cellular senescence induced by aberrant MAD2 levels impacts on paclitaxel responsiveness in vitro
}

\author{
M Prencipe*, , P Fitzpatrick' ${ }^{2}$ S Gorman ${ }^{3}$, M Mosetto $^{3}$, R Klinger ${ }^{4}$, F Furlong', M Harrison ${ }^{5}$, D O'Connor ${ }^{4}$, \\ IB Roninson ${ }^{6}$, J O'Sullivan ${ }^{3}$ and A McCann'
}

'UCD School of Medicine and Medical Science (SMMS), University College Dublin, UCD, Belfield, Dublin 4, Ireland; ${ }^{2}$ UCD School of Public Health and Population Science, University College Dublin, UCD, Belfield, Dublin 4, Ireland; ${ }^{3}$ Centre for Colorectal Disease, St Vincents University Hospital, Dublin 4, Ireland; ${ }^{4}$ School of Biomolecular and Biomedical Science (SBBS), University College Dublin, UCD, Belfield, Dublin 4, Ireland; ${ }^{5}$ Department of Pathology, Mater Misericordiae Hospital, Eccles Street, Dublin 7, Ireland; ${ }^{6}$ Cancer Centre, Ordway Research Institute, Albany, NY, USA

\begin{abstract}
BACKGROUND: The mitotic arrest deficiency protein 2 (MAD2) is a key component of the mitotic spindle assembly checkpoint, monitoring accurate chromosomal alignment at the metaphase plate before mitosis. MAD2 also has a function in cellular senescence and in a cell's response to microtubule inhibitory (MI) chemotherapy exemplified by paclitaxel.

METHODS: Using an siRNA approach, the impact of MAD2 down-regulation on cellular senescence and paclitaxel responsiveness was investigated. The endpoints of senescence, cell viability, migration, cytokine expression, cell cycle analysis and anaphase bridge scoring were carried out using standard approaches.

RESULTS: We show that MAD2 down-regulation induces premature senescence in the MCF7 breast epithelial cancer cell line. These MAD2-depleted (MAD2 $\downarrow$ ) cells are also significantly replicative incompetent but retain viability. Moreover, they show significantly higher levels of anaphase bridges and polyploidy compared to controls. In addition, these cells secrete higher levels of IL-6 and IL-8 representing key components of the senescence-associated secretory phenotype (SASP) with the ability to impact on neighbouring cells. In support of this, MAD2 $\downarrow$ cells show enhanced migratory ability. At $72 \mathrm{~h}$ after paclitaxel, MAD2 $\downarrow$ cells show a significant further induction of senescence compared with paclitaxel naive controls. In addition, there are significantly more viable cells in the MAD2 $\downarrow$ MCF7 cell line after paclitaxel reflecting the observed increase in senescence.

CONCLUSION: Considering that paclitaxel targets actively dividing cells, these senescent cells will evade cytotoxic kill. In conclusion, compromised MAD2 levels induce a population of senescent cells resistant to paclitaxel.

British Journal of Cancer (2009) I II, 1900-1908. doi:I0.1038/sj.bjc.66054l9 www.bjcancer.com
\end{abstract}

(c) 2009 Cancer Research UK

Keywords: mitotic assembly deficient protein 2 (MAD2); cellular senescence; paclitaxel resistance; breast cancer

Cellular senescence can be described as replicative or premature. Telomere shortening results in the former (Harley et al, 1990; Bodnar et al, 1998), whereas oncogene activation (e.g. mutations in K-ras, B-raf, PTEN and NF1) (Courtois-Cox et al, 2008) and stress signals (Shay and Roninson, 2004) such as DNA targeting chemotherapy (doxorubicin or cisplatin) or microtubule inhibitory (MI) chemotherapy (paclitaxel and vincristine) result in the latter. Typically, senescent cells acquire a characteristic flattened and enlarged morphology. In addition, the cytoplasm appears vacuole-rich and granulated due to the expansion of the lysosomal compartment leading to increased expression of the lysosomal $\beta$-galactosidase enzyme, a commonly assessed marker of senescence. These cells also become refractory to apoptosis (Hampel et al, 2004) displaying an altered gene expression profile, with the down-regulation of genes involved in cell proliferation (e.g. Ki-67 and CDC2) and up-regulation of multiple genes with documented growth-inhibitory activity (e.g. p16) (Chang et al, 2002).

*Correspondence: Dr M Prencipe; E-mail: maria.prencipe@ucd.ie Received 8 June 2009; revised 16 September 2009; accepted 12 October 2009
Recently, substantial evidence has identified cellular senescence as an alternative tumour suppressor mechanism to apoptosis (Courtois-Cox et al, 2008). Supporting this function, following oncogene activation, senescent cells can be found in pre-malignant lesions but not in malignant tumours (Collado et al, 2005), identifying an involvement of cellular senescence in preventing tumour progression. However, their presence may also identify a more sinister function in that senescent cells can interfere with their microenvironment, by secreting proteases and mitogenic, antiapoptotic and angiogenic factors, which may promote carcinogenesis in neighbouring cells (Chang et al, 2002). This phenomenon, recently described as the 'senescenceassociated secretory phenotype' (SASP) (Young and Narita, 2009), is gaining considerable research interest. In addition, although it has been documented that cellular senescence is irreversible (Hayflick, 1965), several reports refute this (Beauséjour et al, 2003; Rajaraman et al, 2006). Specifically, Beauséjour et al (2003) have shown that it is possible to revert cellular senescence following inactivation of $\mathrm{p} 53$, if $\mathrm{p} 16$ is concomitantly down-regulated.

The mitotic arrest deficiency protein 2 (MAD2) is an essential component of the mitotic spindle checkpoint pathway and has a crucial function in the transition from metaphase to anaphase by 
delaying anaphase onset, through inhibition of the anaphase promoting complex/cyclosome (APC/C). This ensures that all chromosomes are correctly aligned at the metaphase plate prior to daughter cell segregation (Wang et al, 2002). For this reason, a compromised mitotic spindle assembly checkpoint has an important function in chromosomal instability (CIN) (Yoon et al, 2002), characterised by accelerated rates of chromosomal aberration (Lengauer et $a l, 1998$ ) and resultant aneuploidy (Lengauer et al, 1997). Although the molecular mechanisms underlying a defective mitotic spindle assembly checkpoint and associated CIN in cancer remain elusive, it is notable that cancer cell lines expressing low levels of MAD2 display a defective mitotic checkpoint concomitant with CIN (Li and Benezra, 1996; Percy et al, 2000). Importantly, MAD2 has a crucial function in the malignant transformation of epithelial cells, promoting aneuploidy and predisposing human prostate epithelial cells to carcinogeninduced malignant transformation (To-Ho et al, 2008). This suggests that inactivation of the mitotic spindle assembly checkpoint, through aberrant expression of MAD2, may constitute one of the very initial steps in the malignant progression of human cancer.

Recently, To-Ho et al (2008) have shown a relationship between the cellular expression of a truncated form of the MAD2 protein and increased senescence in human prostate epithelial cells. MAD2 has also been shown to be down-regulated in doxorubicin-induced senescent cells (Chang et al, 2002). Because of its integral function as a cell cycle checkpoint during mitosis, MAD2 is also thought to have a function in paclitaxel resistance (Sudo et al, 2004).

As a chemotherapeutic agent, paclitaxel is increasingly being used as a MI chemotherapy treatment for breast, ovarian and other solid cancers. However, up to a third of patients are not responsive and although several mechanisms have been associated with paclitaxel resistance (McGrogan et al, 2008), reliable molecular markers predicting resistance remain elusive.

In this study, we show a significant association between depleted levels of MAD2 and the induction of cellular senescence, with further senescence induction following paclitaxel treatment in human breast cancer cells. We suggest that MAD2-depleted tumours will not be eradicated by MI therapy exemplified by paclitaxel due to the presence and further induction of a viable senescent cell population capable of interfering with and impacting on the microenvironment through the secretion of transforming growth factors that influence their neighbouring cells (Young and Narita, 2009). Moreover, senescent cells also have the potential to re-enter the cell cycle identifying them as highly chemo-refractory (Beauséjour et al, 2003; Rajaraman et al, 2006).

\section{MATERIALS AND METHODS}

\section{Cell culture and treatments}

MCF-7 cells (ATCC) are an epithelial-like breast adenocarcinoma cell line. Routinely, they were cultured in DMEM medium (Gibco, Carlsbad, CA, USA) supplemented with $10 \%$ fetal bovine serum, $100 \mu \mathrm{lml}^{-1}$ streptomycin and $100 \mathrm{U} \mathrm{ml}^{-1}$ penicillin. HT1080 human fibrosarcoma cells (ATCC) were cultured in MEM medium (Gibco) supplemented with $10 \%$ fetal bovine serum, $100 \mu \mathrm{ml}^{-1}$ streptomycin and $100 \mathrm{U} \mathrm{ml}^{-1}$ penicillin. Derivation of the HT1080 p21-9 cell line carrying $\mathrm{p} 21$ in an isopropyl- $\beta$-Dthiogalactoside (IPTG)-inducible retroviral vector has been described earlier (Chang et al, 1999). The paclitaxel experiments consisted of two groups of cells, one untreated and the other treated with $100 \mathrm{~nm}$ paclitaxel (Sigma-Aldrich, St Louis, MO, USA) over a 72-h period. The IPTG induction of p21 was achieved by treating the cells with $50 \mu \mathrm{M}$ IPTG (Fisher Scientific, Hampton, NH, USA) for 3 days.

\section{Small-interfering RNA transfection}

Small-interfering RNA (siRNA) duplexes targeting MAD2 were purchased from Ambion (Austin, TX, USA). MCF7 transfection was performed with Lipofectamine 2000 following commercial (Invitrogen, Carlsbad, CA, USA) protocols using a $40 \mathrm{~nm}$ final concentration of siRNA. Cells transfected with scramble siRNA duplexes (40 nM Ambion) controlled for any off-target effect of siRNA transfection.

\section{Western blot analysis}

Cells were harvested and washed in PBS and lysed in RIPA lysis buffer (Sigma) containing protease and phosphatase inhibitors. Resultant lysates were cleared by centrifugation at $14000 \mathrm{rpm}$ for $20 \mathrm{~min}$ at $4^{\circ} \mathrm{C}$. Thirty $\mu \mathrm{g}$ of protein were resolved by SDS-polyacrylamide gel electrophoresis and transferred to a nitrocellulose membrane (Amersham, Little Chalfont, UK), which was subsequently blocked in 5\% milk and probed with the anti-MAD2 antibody (1:500, BD Biosciences), anti-Cyclin B1 $(1: 2000$, Cell Signaling Technology, Danvers, MA, USA) or p21 (1:1000, Cell Signaling Technology) overnight at $4{ }^{\circ} \mathrm{C}$. $\beta$-actin antibody $(1: 2000$, Sigma-Aldrich) or alternatively GAPDH ( $1: 1000$, Chemicon International, Temecula, CA, USA), were used as loading controls. The membrane was rinsed in TBS Tween 20 and incubated with horseradish peroxidase-conjugated anti-mouse secondary antibody (Pearse, Waltham, MA, USA) and visualised by an Enhanced Chemiluminescence detection system (Pearse).

Densitometrical analysis was carried out using Scion image analysis (http://www.scioncorp.com), following loading control ( $\beta$-actin) adjustment.

\section{Senescence-associated $\boldsymbol{\beta}$-galactosidase activity}

One hundred and fifty thousand cells were seeded in six-well plates. Following MAD2 depletion and paclitaxel treatment for $72 \mathrm{~h}$, the cells were washed twice with PBS, fixed with $2 \%$ formaldehyde (Sigma) and $0.2 \%$ glutaraldehyde (Sigma-Aldrich) in water for $10 \mathrm{~min}$ and washed again in 2 PBS washes. Cells were stained with X-gal staining solution ( $1 \mathrm{mg} \mathrm{ml}^{-1} \mathrm{X}$-gal, $40 \mathrm{mmoll}^{-1}$ citric acid/sodium phosphate pH $6.0,5 \mathrm{mmoll}^{-1}$ potassium ferricyanide, $5 \mathrm{mmoll}^{-1}$ potassium ferrocyanide, $150 \mathrm{mmoll}^{-1} \mathrm{NaCl}, 2 \mathrm{mmoll}^{-1} \mathrm{MgCl}_{2}$ ) for $24 \mathrm{~h}$ in a $\mathrm{CO}_{2}$ incubator at $37^{\circ} \mathrm{C}$. The cells were subsequently rinsed in two washes of PBS and counted using a phase contrast microscope (Olympus CK2, Shinjuku, Tokyo, Japan). Senescent cells were expressed as a percentage of the total number of cells counted (300 cells per well).

\section{Colony forming assay}

Following MAD2 depletion and paclitaxel treatment, cell proliferation was assessed as described by Franken et al (2006). This assay allows enumeration of the number of colonies formed from a single cell seeded. Briefly, after transfection and drug treatment for $72 \mathrm{~h}$, the cells were trypsinized and 500 cells per well were seeded in six-well plates. After 10 days, the media was removed, the cells were washed in two PBS washes and fixed/stained with a mixture of $6.0 \%$ glutaraldehyde (Sigma-Aldrich) and $0.5 \%$ crystal violet (Avonchem, Macclesfield, UK) solution in water for $30 \mathrm{~min}$ at room temperature. The plates were then rinsed in tap water and left to dry overnight. The colonies were counted and the plate efficiency was calculated as the number of colonies formed/ number of cells seeded $\times 100 \%$.

\section{Cell viability assay}

Ten thousand MCF-7 cells per well were cultured in a 96-well plate and transfected with siRNA targeting MAD2, scramble siRNA or left untransfected. Twenty-four hours after transfection, the cells 
were either treated with $100 \mathrm{~nm}$ of paclitaxel or untreated. Ten thousand HT1080 p21-9 cells per well were cultured in a 96-well plate and either treated with $50 \mu \mathrm{M}$ IPTG for 3 days or left untreated. Subsequently, the two groups of cells $(-/+$ IPTG $)$ were either treated with $100 \mathrm{~nm}$ of paclitaxel or left untreated.

After $72 \mathrm{~h}$, the effect on cell viability was examined by the 3-(4,5)-dimethylthiazol-2-yl-2,5-diphenyltetrazolium bromide (MTT) assay. Fifty $\mu \mathrm{l}$ of 3-(4,5)-dimethylthiazol-2-yl-2,5-diphenyltetrazolium bromide solution ( $5 \mathrm{mg} \mathrm{ml}^{-1}$ in PBS; Sigma-Aldrich) were added to each well and the cells were incubated in a $\mathrm{CO}_{2}$ incubator at $37^{\circ} \mathrm{C}$ for $5 \mathrm{~h}$. Following media removal, the 3-(4,5)-dimethylthiazol-2-yl-2,5-diphenyltetrazolium bromide-formazan formed by metabolically viable cells was dissolved in $200 \mu \mathrm{l}$ of DMSO (Sigma-Aldrich), and the absorbance was measured in a plate reader at $550 \mathrm{~nm}$.

\section{Flow cytometric analysis of the cell cycle}

Cell cycle distribution was measured both in the untreated and paclitaxel treated cells. Cells were harvested after $72 \mathrm{~h}$ of treatment, washed in PBS and fixed for at least $4 \mathrm{~h}$ in $70 \%$ cold methanol. The pellets were then washed twice in PBS Tween 20 and resuspended in PBS. Ribonuclease A $\left(0.2 \mathrm{mg} \mathrm{ml}^{-1}\right.$, Sigma $)$ was added, and the cells were incubated at $37^{\circ} \mathrm{C}$ for $30 \mathrm{~min}$. Propidium iodide ( $50 \mu \mathrm{g} \mathrm{ml}^{-1}$, Sigma) was added and the samples were kept on ice for $30 \mathrm{~min}$ before fluorescence-activated cell sorting (FACS) analysis. Data from 10000 cells were collected. Flow cytometry data were analysed by Summit 4.2 software (Dako Colorado, Inc., Fort Collins, CO, USA).

\section{Anaphase bridge scoring}

MCF-7 cells untransfected or transfected with scramble siRNA or MAD2 siRNA were fixed in $70 \%$ methanol and visualised using haematoxylin staining, which is a nuclear staining, before anaphase bridge enumeration. The number of bridges was expressed as a percentage over the total number of cells counted $(n=2000)$.

\section{Invasion assay}

A total of 50000 cells were incubated in the upper chamber of an innocyte migration assay well (Becton Dickinson, Franklin Lakes, NJ, USA) and allowed to migrate through an $8-\mathrm{mm}$ pore for $24 \mathrm{~h}$. Migrated cells were detached using a detachment buffer provided with the kit and their number was quantified using a cellpermeable fluorescent dye. Fluorescence was read on a plate reader at excitation and emission wavelengths of $485 \mathrm{~nm}$ and $520 \mathrm{~nm}$, respectively. The number of migrated cells was normalised to cell growth levels.

\section{Quantification of pro-inflammatory cytokines using the MSD multi-spot human cytokine assays}

The levels of interleukin-8 (IL-8), IL-1 $\beta$ and IL- 6 were measured in tissue culture media using a four-spot multi-array plate (Meso Scale Discovery, Gaithersburg, Washington DC, USA). The assay was carried out following the protocol provided and read using the Sector Imager 2400. The levels of secretions for each cytokine were normalised to cell proliferation.

\section{Statistical analyses}

Where assumptions could be met, analysis of variance with bonferroni testing for inter-group comparisons was used for the comparison of means in three groups; if not, non-parametric tests were used. The Wilcoxon rank sum test was used for two group comparisons. The SAS programme was used for these analyses.

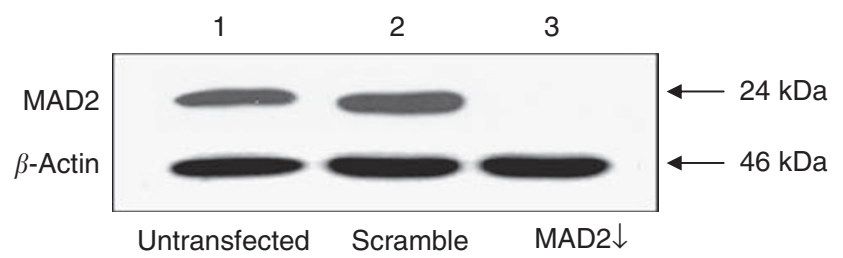

Figure I siRNA MAD2 protein down-regulation in MCF-7 breast cancer cells. Western blot analysis demonstrates a robust reduction of the MAD2 protein in the MAD2 $\downarrow$ cells (lane 3 ) compared with the untransfected (lane I) and scramble (lane 2) controls. $\beta$-actin was used as a loading control.

\section{RESULTS}

\section{MAD2 down-regulation induces cellular senescence in MCF7 breast cancer cells}

Post transfection of the human breast epithelial carcinoma cell line MCF-7 with an siRNA targeting MAD2, western blot analysis showed a robust reduction of the MAD2 protein, compared with the untransfected and scramble controls (Figure 1). Morphologically, MAD2-depleted cells (MAD2 $\downarrow$ ) appeared flattened and enlarged compared with the controls and their cytoplasm typically vacuole-rich typical of senescent cells. Senescence-associated $\beta$-galactosidase activity staining, confirmed that these cells were senescent (Figure 2A, panel c), with a two-fold increase in the percentage of senescence in MAD2 $\downarrow$ cells compared to the untransfected and scramble controls $(P<0.001)$ (Figure $2 \mathrm{~A}$, panel d). Performance of a colony forming assay $96 \mathrm{~h}$ following siRNA transfection (Figure 2B) showed a significantly lower number of colonies $(P<0.05)$ in MAD2 $\downarrow$ cells compared with the untransfected and scramble controls. However, the 3-2,5-diphenyltetrazolium bromide (MTT) viability assay (Figure 2C), showed a similar viability in MAD2 $\downarrow$ cells compared with the controls $(P>0.05)$. The fact that MAD2 down-regulation induced replicative-compromised cells that were viable confirms that these cells had undergone cellular senescence. In addition, confirmatory western blot analysis showed up-regulation of p21, a key marker of cellular senescence (Chang et al, 2000), in the MAD2 $\downarrow$ cells compared with the untransfected and scramble controls (Figure 2D).

\section{MAD2 down-regulation alters cell cycle kinetics inducing anaphase bridge formation}

As expected, FACS analysis showed a highly de-regulated cell cycle, with MAD2 $\downarrow$ cells displaying a significant increase in 4N DNA content $(P<0.0001)$ compared with the scramble and untransfected controls (Figure $3 \mathrm{~A}$ ). To determine whether these were $4 \mathrm{~N}$ cells arrested in mitosis or alternatively tetraploid G1 cells, we assessed the cyclin B1 levels by western blotting (Figure 3B), finding a decrease in cyclin B1 in MAD2 $\downarrow$ cells, indicative of a mitotic slippage. This points to the fact that MAD2 $\downarrow$ cells are not retained in mitosis long enough to allow for efficient chromosomal segregation. This suggests that, as a consequence, aberrant mitosis may occur resulting in anaphase bridge formation. These bridges represent lagging chromosomes, caused by sister chromatids failing to separate completely at the metaphase-anaphase transition. When they do eventually break, one daughter cell can end up with either a gain or a loss of chromosomal material (Saunders, 2003). Significantly in this study, anaphase bridge enumeration showed a three-fold higher percentage of anaphase bridges in the MAD2 $\downarrow$ cells compared with the untransfected and scramble controls $(P \leqslant 0.001)$ (Figure 3C). Moreover, we showed a significantly higher percentage of polyploidy $(P<0.01)$ (Figure $3 \mathrm{D})$ in the MAD2 $\downarrow$ cells compared with controls. 
Down-regulation of MAD2 induces cellular senescence
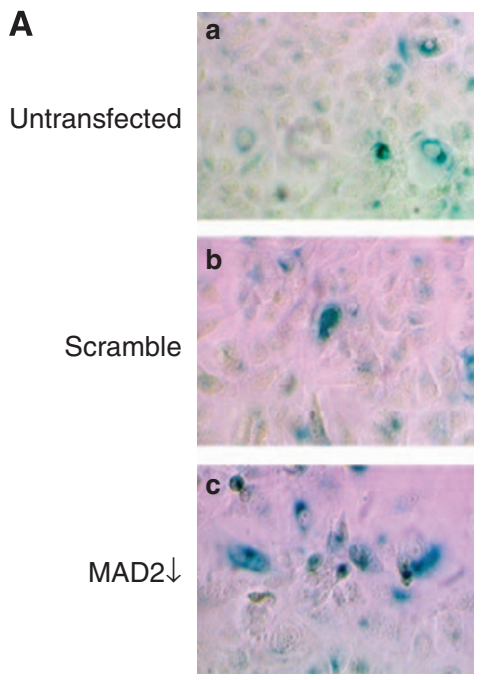

d

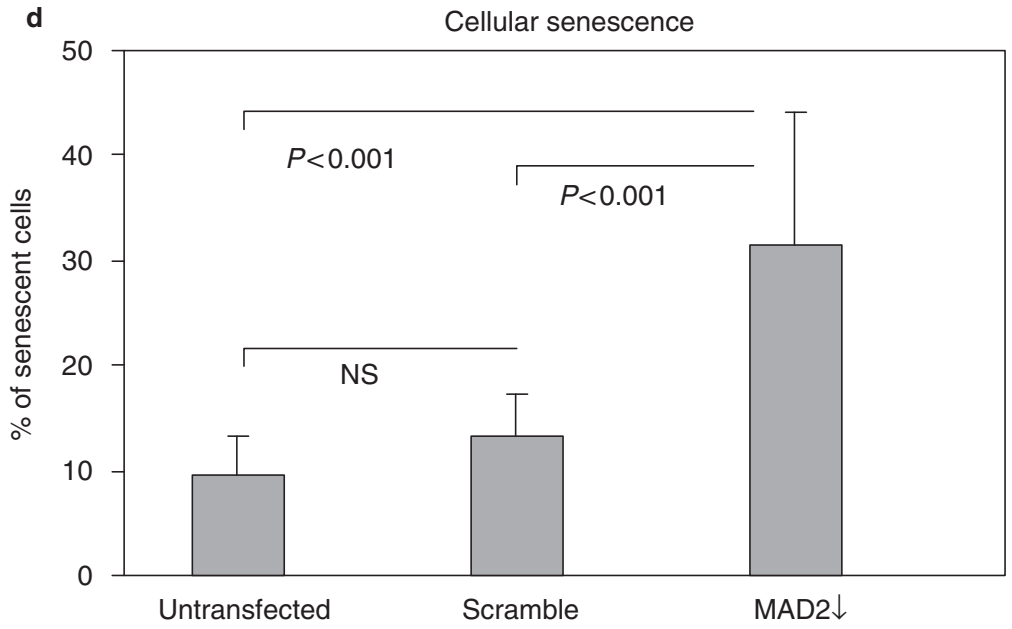

B

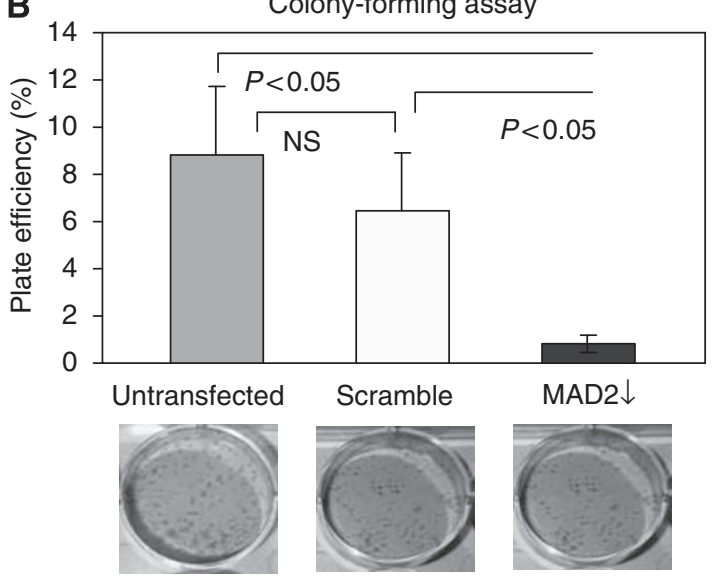

C Cell viability (MTT)

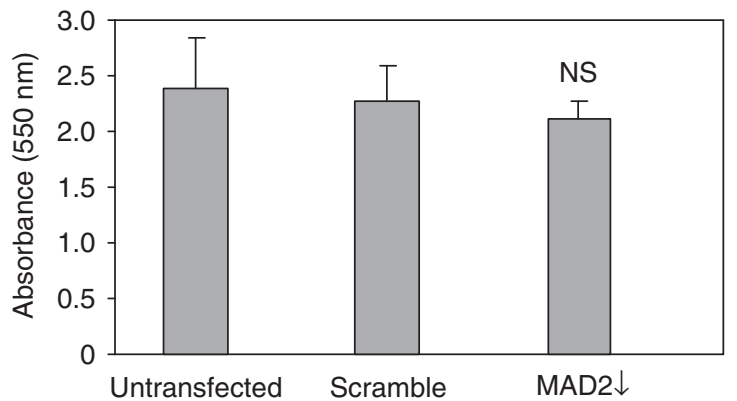

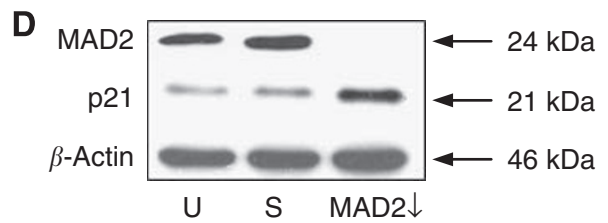

Figure 2 MAD2 down-regulation induces cellular senescence in MCF-7 breast cancer cells. $(\mathbf{A}) 15 \times 10^{4}$ cells were seeded in each well of a six-well plate. They were either left untransfected or transfected with either MAD2 siRNA or scramble siRNA. Post siRNA reduction of MAD2, morphological changes were evident with MAD2-depleted (MAD2 $\downarrow$ ) cells appearing flattened and enlarged and more vacuole-rich, compared with the untransfected and scramble controls. SA- $\beta$-galactosidase staining (panels $\mathrm{a}-\mathrm{c}$ ) shows a significantly higher percentage of senescent cells in the MAD2 $\downarrow$ cells compared with the untransfected and scramble controls $(P<0.00 \mathrm{I}$ ) (panel d). (B) Plate efficiency percentage (number of colonies formed/number of cells seeded) $\times 100 \%$, in MAD2 $\downarrow$ cells compared with the untransfected and scramble controls. The colony count showed a significant lower number of colonies in MAD2 $\downarrow$ cells, compared with the controls $(P<0.05)$. (C) The MTT assay showed the same viability for untransfected, scramble and MAD2 $\downarrow$ cells $(P>0.05)$. (D) Western blotting analysis showed up-regulation of the p2I senescent marker following siRNA down-regulation of MAD2 compared with the untransfected and the scramble controls. $\beta$-actin was used as a loading control.

\section{MAD2 down-regulation alters the protein secretion of cells and enhances cell migration}

Having found a direct association between MAD2 down-regulation and cellular senescence induction and considering the known ability of senescent cells to secrete inflammatory cytokines capable of interfering with their microenvironment (Young and Narita, 2009), we performed an ELISA test to look at the expression levels of IL-1 $\beta$, IL-6 and IL-8 following MAD2 down-regulation. Intriguingly, we have found a three-fold increase in IL-6 in the MAD2 $\downarrow$ cells compared with the scramble control $(P<0.01)$ and nine-fold compared with the untransfected control $(P<0.001)$ (Figure 4A, panel a). IL-8 expression was significantly higher in the MAD2 $\downarrow$ cells compared with the untransfected control $(P=0.01)$ but did not reach significance in comparison with the scramble control, despite showing a trend towards higher levels in the MAD2 $\downarrow$ cells $(P=0.06)$ (Figure $4 \mathrm{~A}$, panel $\mathrm{b})$. Moreover, IL-1 $\beta$ did not show a statistically significant increase in MAD2 $\downarrow$ cells compared with the controls. Interestingly, a migration assay showed a statistically significant higher migration ability of the MAD2 $\downarrow$ cells compared with the untransfected $(P<0.01)$ and scramble $(P<0.05)$ controls (Figure $4 \mathrm{~B})$. 
A

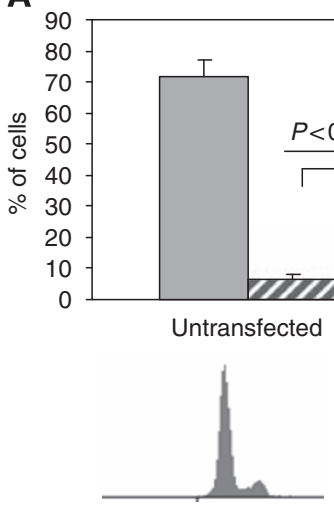

Cell cycle

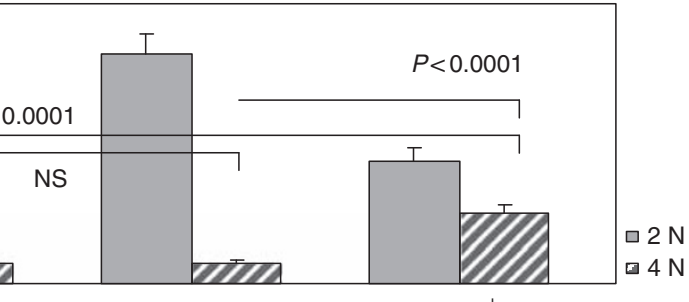

B

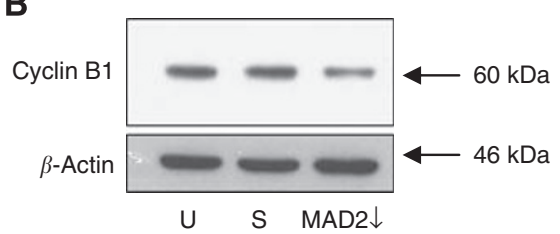

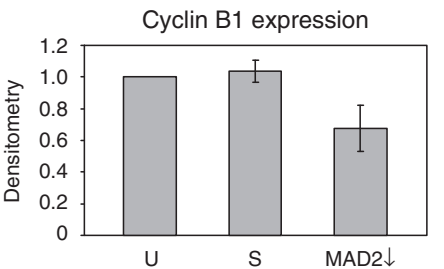

C
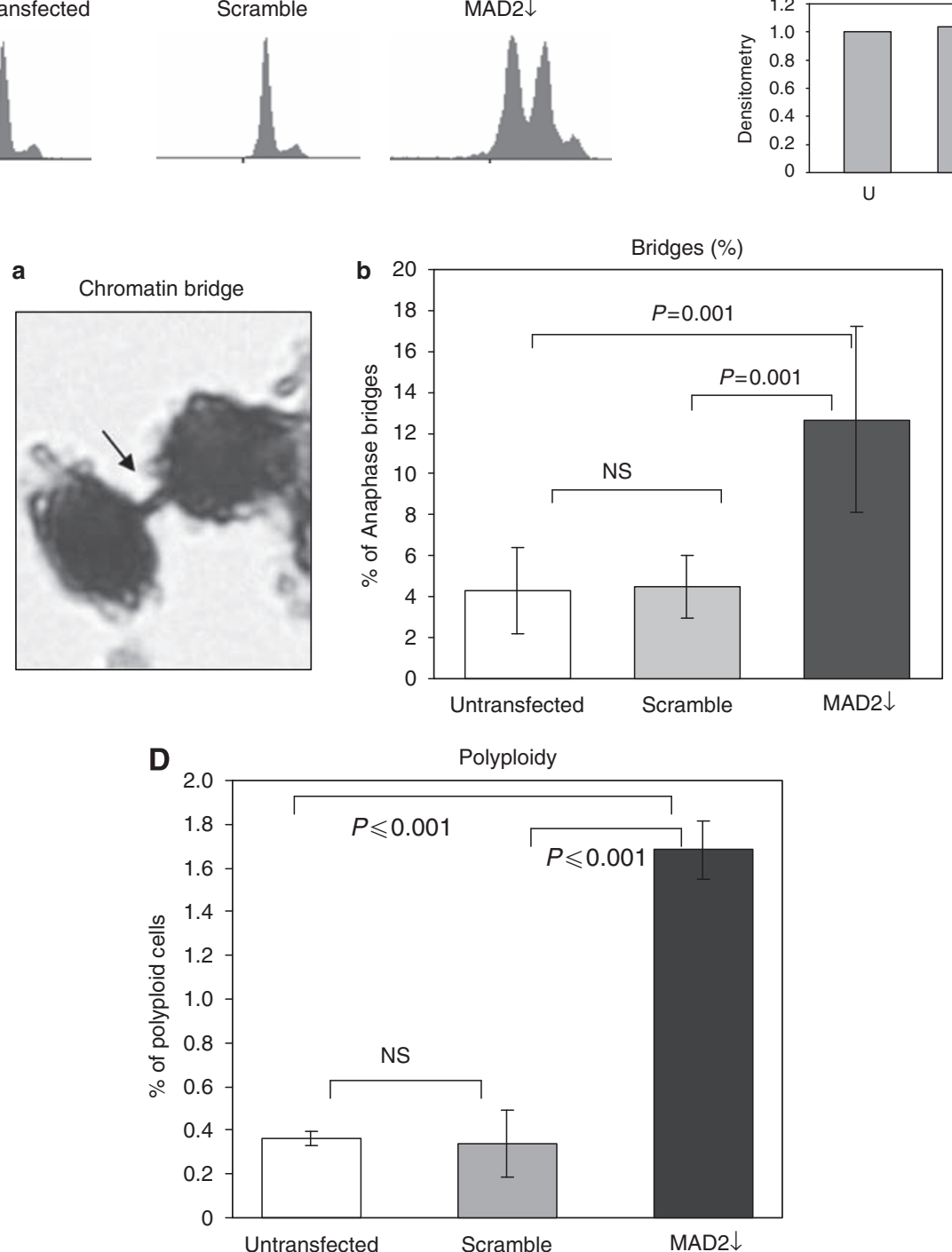

Figure 3 MAD2 down-regulation alters cell cycle kinetics inducing anaphase bridge formation. (A) Following MAD2 siRNA down-regulation, the cell cycle appeared highly de-regulated, showing less cells with $2 \mathrm{~N}$ DNA content $(P<0.000 \mathrm{I})$ and more in $4 \mathrm{~N}(P<0.000 \mathrm{I})$. (B) Western blotting analysis of cyclin $\mathrm{B} I$ shows a significant decrease of this protein in the MAD2 $\downarrow$ cells compared with the untransfected and scramble controls. (C) Anaphase bridges were visualised using haematoxylin staining, which is a nuclear staining able to highlight only the nuclear content (panel a). MAD2 $\downarrow$ cells demonstrate a threefold higher percentage of anaphase bridges compared with the untransfected and scrambled controls $(P \leqslant 0.00 \mathrm{I})$ (panel b). (D) MAD2 down-regulation also results in a higher percentage of polyploid cells $(>4 \mathrm{~N})$ than the control lines $(P \leqslant 0.00 \mathrm{I})$. Flow cytometry data were analysed by Summit 4.2 software. Mean values were compared using the $t$-test assuming equal variances.

\section{MAD2 reduction further exacerbates the induction of viable senescent cells after paclitaxel treatment}

Due to the observed association between MAD2 down-regulation and the induction of cellular senescence, we investigated how MAD2 depletion would impact on the MAD2 $\downarrow$ cells' response to paclitaxel. Phase contrast images captured $72 \mathrm{~h}$ post paclitaxel treatment, showed that MAD2 $\downarrow$ cells remained attached to the flask with many displaying typical senescence morphology (Figure $5 \mathrm{~A}$, panel $\mathrm{f}$ ). This was in stark contrast to the untransfected and scramble controls (Figure 5A, panels $\mathrm{d}$ and e), which displayed a rounded shape, indicative of G2/M arrest or early apoptosis (data not shown) with eventual detaching from the flask; the expected paclitaxel response. The SA- $\beta$-galactosidase staining following paclitaxel treatment, showed not only a significant increase in cellular senescence in MAD2 $\downarrow$ cells compared with the 
A

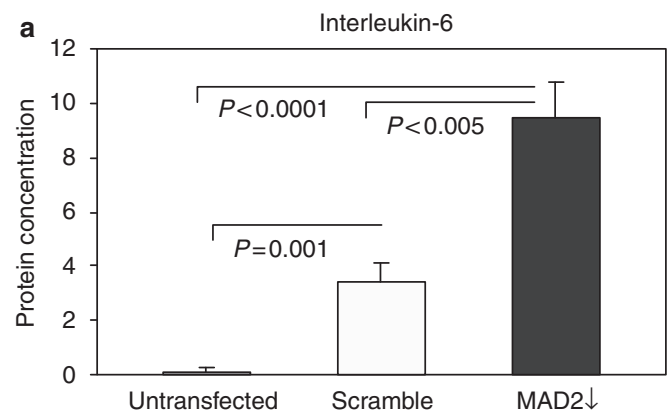

b

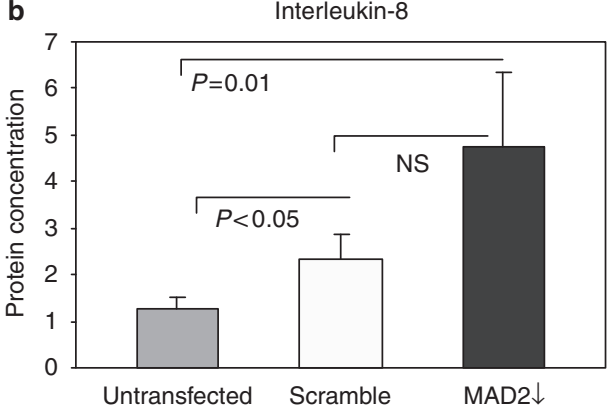

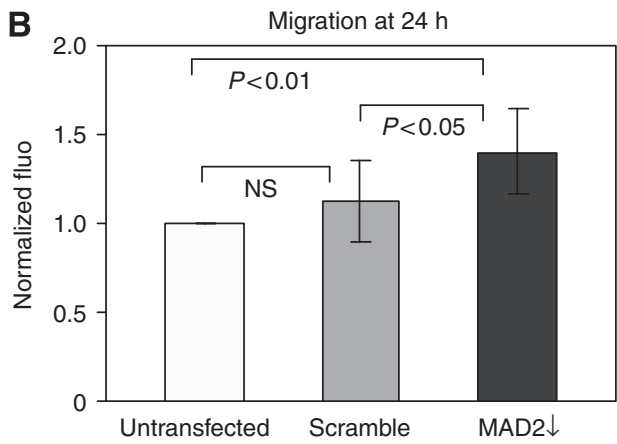

Figure 4 MAD2 down-regulation alters the protein secretion of cells and enhances cell migration. (A) (Panel a) ELISA analysis shows a three-fold increase in IL-6 levels in the MAD2 $\downarrow$ cells compared with the scramble $(P<0.005)$ and the untransfected $(P<0.00$ I $)$ controls. (Panel b) MAD2 $\downarrow$ cells show a higher expression of IL-8 compared with the controls. However, this increase reaches statistical significance only in comparison with the untransfected control $(P=0.0 \mathrm{I})$. (B) MAD2 $\downarrow$ cells show a higher migration ability compared with the untransfected $(P<0.0 \mathrm{I})$ and scramble $(P<0.05)$ controls. Mean values were compared using the $t$-test assuming equal variances.

untransfected and scramble controls $(P<0.0001)$ (Figure 5B), but significantly, a further induction of senescence in the paclitaxel treated MAD2 $\downarrow$ cells compared with the paclitaxel naive cultures $(P<0.05)$. The MTT assay, showed more viable cells $(P<0.0001)$ after paclitaxel, in the MAD2 $\downarrow$ cells compared with the untransfected and scramble controls (Figure 5C), whereas no difference in viability was displayed in the MAD2 $\downarrow$ cells before and after paclitaxel treatment. In support of this observation, we performed a colony forming assay after $72 \mathrm{~h}$ of paclitaxel treatment, seeding 10000 cells per well for each condition (untransfected, scramble and MAD2 $\downarrow$ ). In parallel, we also seeded cells for SA- $\beta$ galactosidase staining. After 10 days, although there was no colony formation in any group, there were demonstrably more viable cells in the MAD2 $\downarrow$ well, compared with the untransfected and scramble controls (Figure 5D, panel a). Moreover, the SA- $\beta$ galactosidase staining displayed an increase in cellular senescence in MAD2 $\downarrow$ cells compared with the untransfected and scramble controls (Figure 5D, panels $b-d$ ). These results confirm that the difference in viability in the MAD2 $\downarrow$ cells post paclitaxel treatment, shown by both the MTT assay and by the colony forming assay, was due to senescent cells evading paclitaxelinduced death.

To further support the direct link between cellular senescence and resistance to paclitaxel, we used the human HT1080 p21-9 fibrosarcoma cell line, carrying an IPTG-induced expression cassette, able to drive p21 up-regulation. The cyclin-dependent kinase inhibitor, p21 Waf/Cip1/Sdi1 , is a key negative regulator of cell cycle progression and its up-regulation has been associated with cellular senescence induction (Chang et al, 2000).

We have shown that up-regulation of $\mathrm{p} 21$, in these cells, is able to induce MAD2 down-regulation (Figure 6A) and also an increase in cellular senescence (Figure 6B). An MTT assay showed no difference in viability between the HT1080 p21-9 cells with or without IPTG. This is in agreement with the fact that senescent cells show the same viability as normal cells. However, when we treated the HT1080 p21-9 cells with paclitaxel, a significant difference in viability $(P<0.001)$ was detected between the cells that had been induced with IPTG to become senescent, compared with the cells that had not been IPTG induced. Specifically, the latter showed a 2.5-fold increase in cell death in comparison to the former (Figure 6C).

\section{DISCUSSION}

Several reports have suggested a function for MAD2 in the onset of premature senescence. Specifically, MAD2 is down-regulated in doxorubicin-induced senescent cells (Chang et al, 2002), its expression is reduced in vitro in aging pig oocytes (Ma et al, 2005) and the expression of a truncated form of MAD2 is associated with cellular senescence in human prostate epithelial cells (To-Ho et al, 2008). In this study, we have found the first link between the induction of cellular senescence and MAD2 down-regulation in human breast cancer cells. Following down-regulation of MAD2, the MCF-7 breast cancer cells underwent a dramatic change in their morphology, cell cycle and ability to proliferate; hallmarks of cellular senescence. At $72 \mathrm{~h}$ following MAD2 down-regulation, $\sim 30 \%$ of the cells showed features of senescence, a result strikingly similar to that reported by To-Ho et al in human prostate epithelial cells, following expression of a truncated form of MAD2 protein. These comparative results, in cells of different lineages, corroborate the hypothesis that aberrant MAD2 levels have a significant function in the induction of cellular senescence.

The induction of this form of cellular fate is biologically significant as senescent cells are able to modify their microenvironment by the secretion of transforming factors (Kuilman and Peeper, 2009; Young and Narita, 2009). Interestingly, following MAD2 down-regulation, we have shown an aberrant increased secretion of IL- 6 and IL- 8 , which have been identified as key components of the SASP (Coppé et al, 2008), having a crucial function in EMT induction. Relevantly, this result was mirrored by the increased ability of migration displayed by MAD $2 \downarrow$ cells. 
A

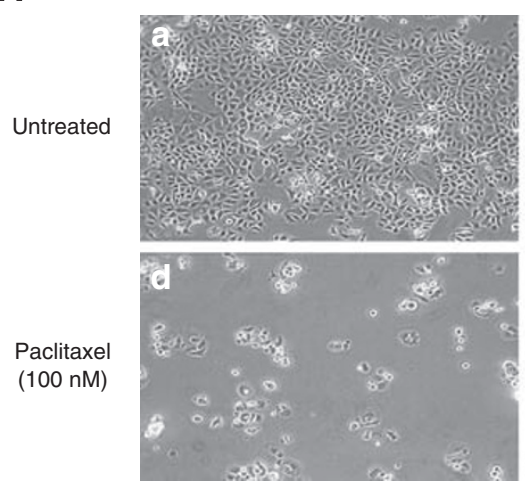

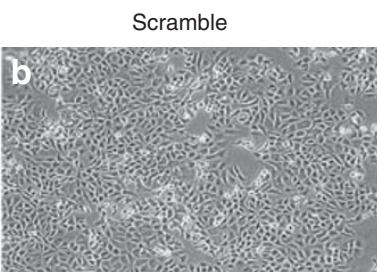

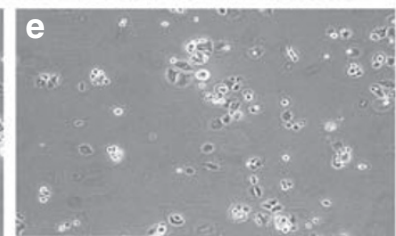

C

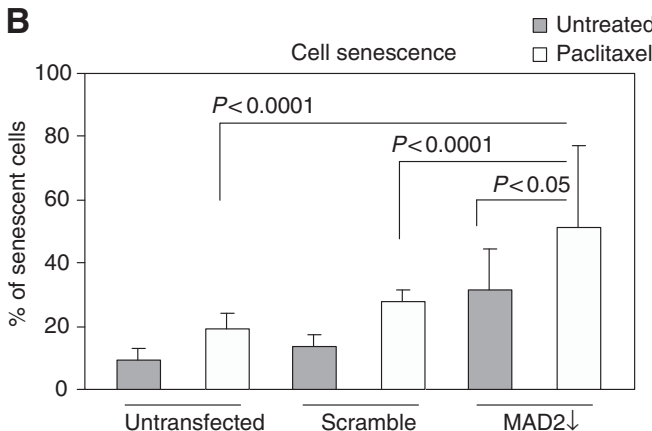

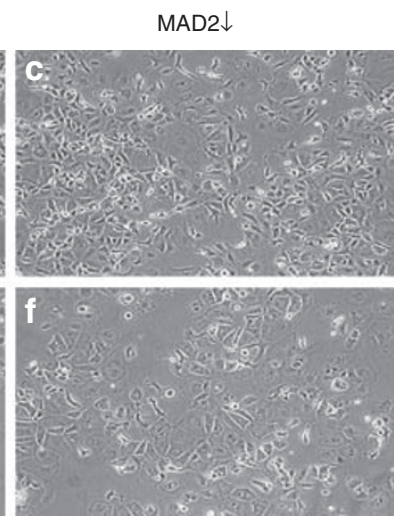

D
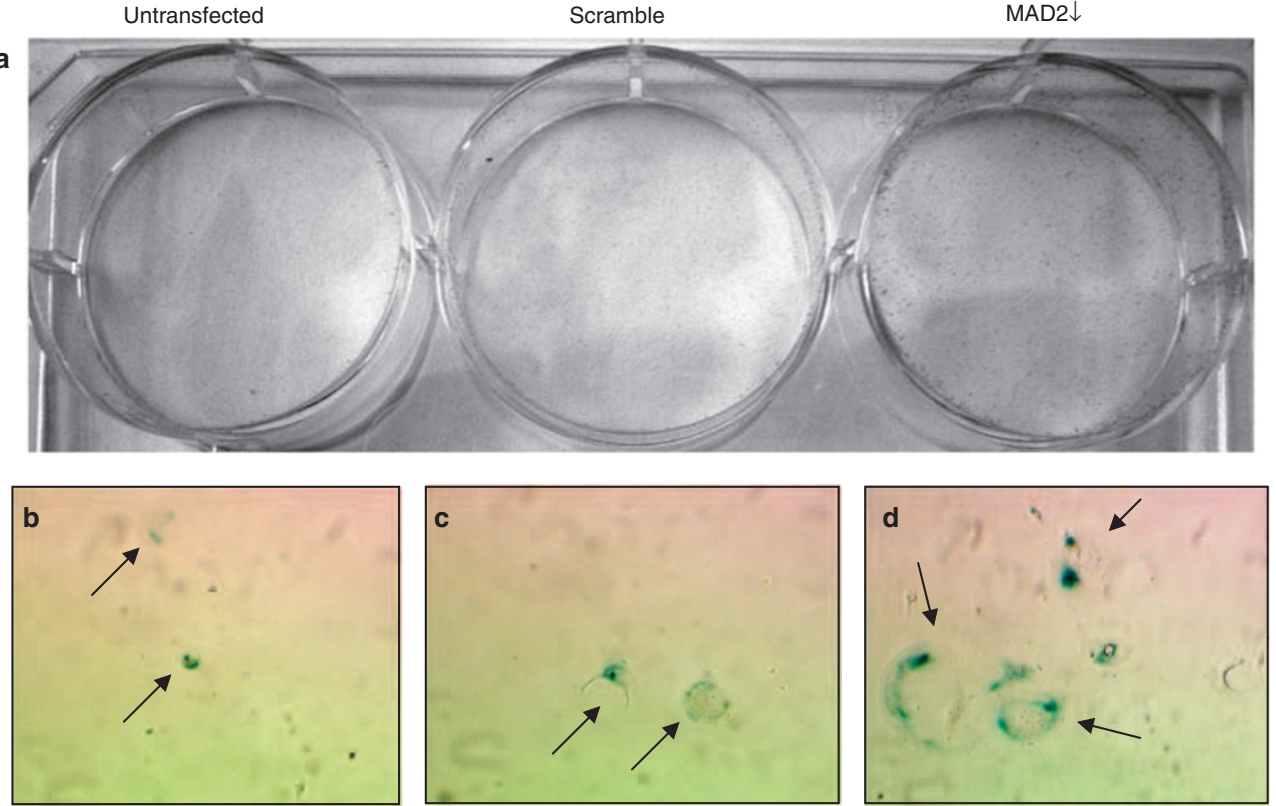

Figure 5 MAD2 down-regulation induces paclitaxel resistance through cellular senescence induction in MCF7 cells. (A) $15 \times 10^{4}$ cells were seeded in each well of a six-well plate. They were either left untransfected or transfected with either MAD2 siRNA or scramble siRNA. Twenty-four hours following transfection, they were either treated with $100 \mathrm{~nm}$ of paclitaxel or left untreated. Phase contrast images captured $72 \mathrm{~h}$ post paclitaxel treatment showed that MAD2 $\downarrow$ cells remained attached to the flask with many displaying typical senescence morphology (panel f), contrasting dramatically with the untransfected and scramble controls (Panels $d$ and $e$ ), which display a rounded shape eventually detaching from the flask. (B) The SA- $\beta$-galactosidase staining post paclitaxel treatment shows an increase in cellular senescence in the MAD2 $\downarrow$ cells compared with the untransfected and scramble controls $(P<0.000 \mathrm{I})$. Moreover, a significantly higher percentage of senescent cells in the MAD2 $\downarrow$-depleted cells was observed $(P<0.05)$ compared with the MAD2 $\downarrow$ untreated cells. $(\mathbf{C})$ The MTT assay shows a significantly higher $(P<0.000 \mathrm{I})$ percentage of viability in the MAD2 $\downarrow$ cells post paclitaxel treatment compared with the untransfected and scramble controls. (D) Post transfection and I00 nM paclitaxel treatment for $72 \mathrm{~h}$, cells were trypsinized and 10000 cells were seeded in each well and cultured for 10 days. (Panel a) Crystal violet staining showed significantly more viable cells in the MAD2 $\downarrow$ well compared with the untransfected and scramble controls. (Panels b-d) Phase contrast pictures at $10 \times$ magnification show more senescence cells (arrows) in the MAD2 $\downarrow$ well compared with the untransfected and scramble controls. 


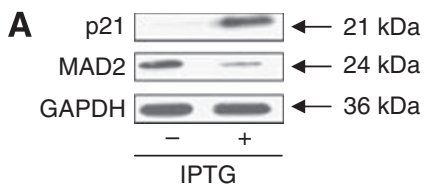

B
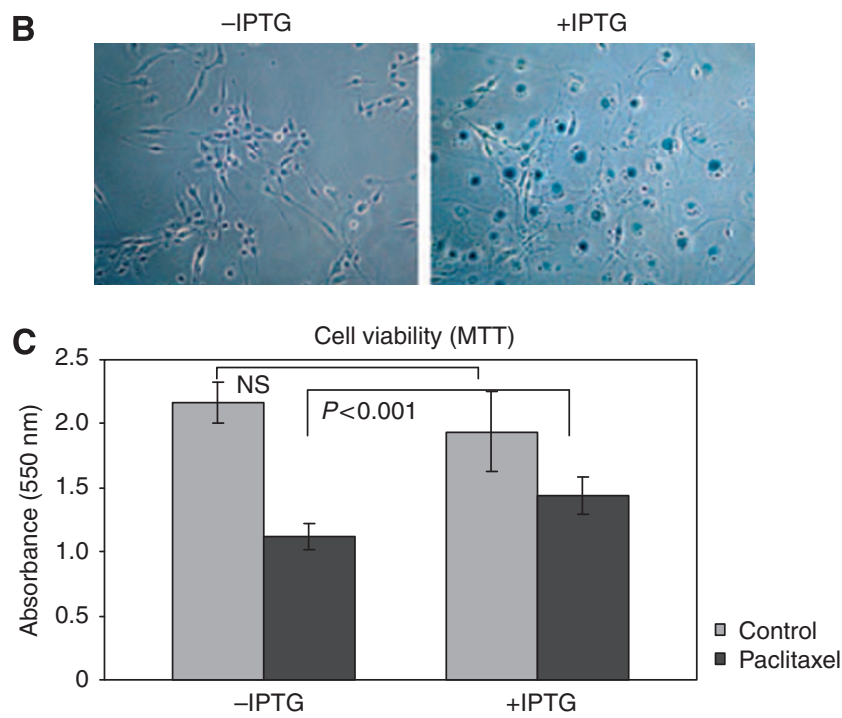

Figure $6 \mathrm{p} 2 \mathrm{I}$ induction of cellular senescence induces paclitaxel resistance in HTI080 p2 I-9 human fibrosarcoma cells. (A) Western blot analysis shows down-regulation of the MAD2 protein following upregulation of $\mathrm{P} 21$, induced by IPTG. GAPDH was used as a loading control. (B) $\beta$-galactosidase staining shows an increase in senescent cells following IPTG induction of $\mathrm{p} 2 \mathrm{I}$ and concomitant MAD2 down-regulation. (C) The MTT cell viability assay shows the same viability in the HTI080 p2I-9 cells before paclitaxel treatment, regardless of $\mathrm{p} 2 \mathrm{I}$ induction by IPTG. However, a significant higher viability is displayed by the IPTG-treated cells when compared with the IPTG non-treated cells, after paclitaxel $(P<0.00 \mathrm{I})$.

Our data also show a significant increase in anaphase bridge formation and a higher percentage of polyploid cells post MAD2 down-regulation. Significantly, as reviewed by Storchova and Pellman (2004), polyploid cells are highly unstable, potentially contributing to aneuploidy in cancer, whereas anaphase bridge formation may result in severe aneuploidy, clearly associated with CIN in cancer (Lengauer et al, 1997). The fact that MAD2 defective cells are more prone to $\mathrm{CIN}$, through anaphase bridge formation, is particularly interesting from a treatment point of view. In fact, it has been shown that the presence of CIN in tumours before drug exposure, seems to predict for intrinsic taxane resistance (Swanton et al, 2006).

Following on from these observations, we investigated what effect premature senescence (induced by aberrant MAD2 levels) had on paclitaxel responsiveness. SA- $\beta$-galactosidase staining showed that, after paclitaxel treatment, $50 \%$ of the population

\section{REFERENCES}

Beauséjour CM, Krtolica A, Galomi F, Narita M, Lowe SW, Yaswen P, Campisi J (2003) Reversal of human cellular senescence: role of the p53 and p16 pathway. EMBO J 16: $4212-4222$

Bodnar AG, Ouellette M, Frolkis M, Holt SE, Chiu CP, Morin GB, Harley CB, Shay JW, Lichtsteiner S, Wright WE (1998) Extension of life span by introduction of telomerase into normal human cells. Science 279: 349-352

Chang BD, Xuan Y, Broude EV, Zhu H, Schott B, Fang J, Roninson IB (1999) Role of p53 and p21waf1/cip1 in senescence-like terminal proliferation arrest induced in human tumor cells by chemotherapeutic drugs. Oncogene 18(34): $4808-4818$

Chang BD, Watanabe K, Broude EV, Fang J, Poole JC, Kalinichenko TV, Roninson IB (2000) Effects of $221^{\text {Waf1/Cip } 1 / \text { dil }}$ on cellular gene had undergone senescence in the MAD2 $\downarrow$ cells. This percentage was higher than that seen in the paclitaxel naive MAD2 $\downarrow$ cells $(30 \%)$, suggesting that paclitaxel had in fact exacerbated the induction of cellular senescence. We also found that there were significantly more viable cells in the MAD2 $\downarrow$ population after paclitaxel, reflecting the observed increase in cellular senescence. Most importantly, the fact that the colony forming assay did not show any colony formation after paclitaxel treatment for either of the three groups (untransfected, scramble and MAD2 $\downarrow$ ), is not an indication that the cellular fate was similar in each of the three groups. In fact, the colony forming assay can only give information about the ability of the cells to proliferate but cannot discriminate the cellular mechanism underlying this lack of proliferation, which in our case appears to be apoptosis in the two control groups with cellular senescence in the MAD2 $\downarrow$ cells.

Further support of our findings comes from the HT1080 p21-9 data, where we have shown a 2.5 -fold higher viability $(P<0.001)$ post paclitaxel treatment, on senescence induction through upregulation of $\mathrm{p} 21$ and consequent MAD2 down-regulation.

The ominous function that senescent cells may have in the chemotherapeutic arena lies in the fact that firstly, recent evidence has shown that senescent cells can interfere with their microenvironment, by secreting transforming factors, which may promote carcinogenesis in neighbouring cells (Chang et al, 2002), mirrored by our cytokine data. Secondly, although it is widely documented that cellular senescence is irreversible (Hayflick, 1965), other data refute this (Beauséjour et al, 2003; Rajaraman et al, 2006). Intriguingly, Rajaraman et al describe a novel process of cell division occurring only in senescent cells, termed neosis resulting in multiple smaller cells (Raju cells), which in turn are able to re-enter the cell cycle giving rise to a more aggressive cell population.

In summary, we have shown that down-regulation of MAD2, a feature common to many tumour cell lineages (Takahashi et al, 1999; Wang et al, 2000, 2002; Fung et al, 2007) induces cellular senescence in up to a third of the cell population. Due to the fact that paclitaxel targets actively dividing cells, these cells will evade cytotoxic-induced death. Corroborating this, we have found no decrease in cell viability post paclitaxel treatment in the MAD2 $\downarrow$ cells and a significant increase in cell senescence. Our results warrant further investigation as paclitaxel treatment may not be beneficial and even deleterious to those tumours displaying low levels of MAD2.

\section{ACKNOWLEDGEMENTS}

We thank the Irish Research Council for Science, Engineering and Technology (IRCSET). This material is based on works supported by the Irish Cancer Society under Grant CRF08FUR and supported by the Health Research Board Ireland (GrantRP/2006/80). Maria Prencipe was partially supported by funding from the College of Life Sciences (CLS) Epigenetics Research Group UCD (CERGU), University College Dublin, UCD, Belfield Dublin 4, Ireland. expression: implications forcarcinogenesis, senescence, and age-related diseases. PNAS USA 97(8): 4291-4296

Chang BD, Swift ME, Shen M, Fang J, Broude EV, Roninson IB (2002) Molecular determinants of terminal growth arrest induced in tumor cells by a chemotherapeutic agent. PNAS 99: 389-394

Collado M, Gil J, Efeyan A, Guerra C, Schuhmacher AJ, Barradas M, Benguría A, Zaballos A, Flores JM, Barbacid M, Beach D, Serrano M (2005) Senescence in premalignant tumours. Nature 436: 642

Coppé JP, Patil CK, Rodier F, Sun Y, Munoz DP, Goldstein J, Nelson PS, Desprez PY, Campisi J (2008) Senescence-associated secretory phenotypes reveal cell-nonautonomous functions of oncogenic RAS and the p53 tumour suppressor. PLoS Biol 6: e301 
Courtois-Cox S, Jones SL, Cichowsky K (2008) Many roads lead to oncogene-induced senescence. Oncogene 27: 2801-2809

Franken NAP, Rodermond HM, Stap J, Haveman J, van Bree C (2006) Clonogenic assay of cells in vitro. Nat Protoc 1: 2315-2319

Fung KL, Cheung HW, Wong HL, Yuen HF, Ling MT, Chan KW, Wong YC, Cheung AL, Wang X (2007) MAD2 expression and its significance in mitotic checkpoint control in testicular germ cell tumour. Biochem Biophys Acta 1773: $821-832$

Hampel B, Malisan F, Niederegger H, Testi R, Jansen-Durr P (2004) Differential regulation of apoptotic cell death in senescent human cells. Exp Gerontol 39: $1713-1721$

Harley CB, Futcher AB, Greider CW (1990) Telomeres shorten during aging of human fibroblast. Nature 345: $458-460$

Hayflick L (1965) The limited in vitro life time of human diploid cell strains. Exp Cell Res 37: 614-636

Kuilman T, Peeper DS (2009) Senescence-messaging secretome; SMS-ing cellular stress. Nat Rev Cancer 9(2): 81 -94

Lengauer C, Kinzler K W, Vogelstein B (1997) Genetic instability in colorectal cancer. Nature 386: $623-627$

Lengauer C, Kinzler K W, Vogelstein B (1998) Genetic instability in human cancer. Nature 396: 643-649

Li Y, Benezra R (1996) Identification of a human mitotic checkpoint gene: hsMAD2. Science 274: 246-248

Ma W, Zhang D, Hou Y, Li YH, Sun QY, Sun XF, Wang WH (2005) Reduced expression of MAD2 BCL2 MAP kinase activity in pig oocytes after in vitro aging are associated with defects in sister chromatid segregation during meiosis II embryo fragmentation after activation. Biol Reprod 72: $373-383$

McGrogan BT, Gilmartin B, Carney DN, McCann A (2008) Taxanes, microtubules and the chemoresistant breast cancer. Biochim Biophys Acta 1785: $96-132$

Percy MJ, Myrie KA, Neeley CK, Azim JN, Ethier SP, Petty EM (2000) Expression and mutational analysis of the human MAD2L1 gene in breast cancer cells. Genes Chromosomes Cancer 29: $356-362$

Rajaraman R, Guernsey DL, Rajaraman MM, Rajaraman SR (2006) Stem cells, senescence, neosis and self-renewal in cancer. Cancer Cell Int 8: 6-25

Saunders W (2003) Bridging mitotic defects and clinical diagnoses. Cancer Biol Ther 2: $253-255$

Shay JW, Roninson IB (2004) Hallmarks of senescence in carcinogenesis and cancer therapy. Oncogene 23: 2919-2933

Storchova Z, Pellman D (2004) From polyploidy to aneuploidy, genome instability and cancer. Nat Rev Mol Cell Biol 5: 45-54

Sudo T, Nitta M, Saya H, Ueno NT (2004) Dependence of Paclitaxel sensitivity on a functional spindle assembly checkpoint. Cancer Res 64: 2502-2508

Swanton C, Tomlinson I, Downward J (2006) Chromosomal instability, colorectal cancer and taxane resistance. Cell Cycle 5(8): 818-823

Takahashi T, Haruki N, Nomoto S, Masuda A, Saji S, Osada H, Takahashi T (1999) Identification of frequent impairment of the mitotic checkpoint and molecular analysis of mitotic checkpoint genes, hsMAD2 and p55CDC, in human lung cancers. Oncogene 18: 4295-4300

To-Ho KW, Cheung HW, Ling MT, Wong YC, Wang X (2008) Oncogene 27: $347-357$

Wang X, Jin DY, Ng RW, Feng H, Wong YC, Cheung AL, Tsao SW (2002) Significance of MAD2 expression to mitotic checkpoint control in ovarian cancer cells. Cancer Res 62: $1662-1668$

Wang X, Jin DY, Wong YC, Cheung AL, Chun AC, Lo AK, Liu Y, Tsao SW (2000) Correlation of defective mitotic checkpoint with aberrantly reduced expression of MAD2 protein in nasopharyngeal carcinoma cells. Carcinogenesis 21: 2293-2297

Young AR, Narita M (2009) SASP reflects senescence. EMBO Rep 10(3): $228-230$

Yoon DS, Wersto RP, Zhou W, Chrest FJ, Garret ES, Kwon TK, Gabrielson E (2002) Variable levels of chromosomal instability and mitotic spindle checkpoint defects in breast cancer. Am J Pathol 161: $391-397$ 